\title{
Síndrome distérmica (hipertermia) em bovinos associada à intoxicação por Claviceps purpurea ${ }^{1}$
}

\author{
Marcia R. S. Ilha ${ }^{2}$, Franklin Riet-Correa ${ }^{3}$ e Claudio S. L. Barros ${ }^{4}$
}

\begin{abstract}
Ilha M. R. S., Riet-Correa F. \& Barros C. S. L. 2001. [Dysthermic syndrome (hyperthermia) in cattle associated with poisoning by Claviceps purpureal Síndrome distérmica (hipertermia) em bovinos associada à intoxicação por Claviceps purpurea. Pesquisa Veterinária Brasileira 21(2):81-86. Depto Patologia, Universidade Federal de Santa Maria, 97105900 Santa Maria, RS, Brazil. E-mail: ilha76@mailcity.com

Three outbreaks of dysthermic syndrome (hyperthermia) associated with poisoning by Claviceps purpurea are described in dairy cattle in the State of Rio Grande do Sul, Brazil. During the summer of 1999/2000, 37 cattle out of $66(56 \%)$ were affected up to 3 months after the introduction of a new batch of feed contaminated by this fungus. The main clinical signs included pyrexia, dull, rough and long hair coats, intense salivation, difficult respiration with open mouth and, in some cases, with the tongue protruding from the mouth. Affected cattle sought shade or remained within water ponds. There was a $10-30 \%$ reduction in feed intake and loss of weight. Reduction in milk yield was $30-50 \%$. Clinical signs were more intense during daytime, and their intensity were directly proportional to the environmental temperature. Necropsy findings in one necropsied cow included mild pulmonary emphysema, mainly in dorsal aspect of the diaphragmatic lobes. Histollogically, there were moderate hypertrophy of the smooth muscle layer of bronchioles, rupture of alveolar septae with resulting club-shaped alveolar stumps. Sclerotia of $C$. purpurea were found in the ration fed to cattle in the three farms where the outbreaks occurred. Affected cattle recovered approximately 60 days after feeding the contaminated ration was discontinued. Diagnosis was based on epidemiological data, clinical signs, presence of sclerotia of $C$. purpurea in the feed of affected cattle, necropsy and histopathological findings. The pathogenesis, clinical signs and pathology of dysthermic syndrome are discussed and compared to other reports on this condition.
\end{abstract}

INDEX TERMS: Dysthermic syndrome, hyperthermia, Claviceps purpurea, mycotoxicosis, diseases of cattle.

RESUMO.- Descrevem-se três surtos de síndrome distérmica (hipertermia) associada à intoxicação por Claviceps purpurea, em bovinos de leite durante o verão de 1999-2000, em três estabelecimentos do Rio Grande do Sul. De um total de 66 bovinos que ingeriram a ração contaminada com o fungo, 37 (56\%) adoeceram até 3 meses após a introdução da ração contaminada. Os principais sinais clínicos foram temperatura retal

\footnotetext{
${ }^{1}$ Aceito para publicação em 8 de maio de 2001.

${ }^{2}$ Programa de Pós-graduação em Medicina Veterinária, Mestrado, Universidade Federal de Santa Maria (UFSM), 97105-900 Santa Maria, RS. E-mail: ilha76@mailcity.com.br

${ }^{3}$ Laboratório Regional de Diagnóstico, Universidade Federal de Pelotas, 96010-900 Pelotas, RS. E-mail: riet@ufpel.tche.br

${ }^{4}$ Depto Patologia, UFSM, 97105-900 Santa Maria, RS. E-mail: cslb@sm.conex.com.br
}

elevada, pêlos compridos, longos e sem brilho, salivação intensa, respiração ofegante, com a boca aberta e, em alguns casos, com a língua para fora da cavidade oral. Os animais acometidos procuravam sombra ou permaneciam dentro d'água. Houve diminuição de 10 a $30 \%$ no consumo de alimentos e perda de peso. A redução na produção de leite foi de 30 a $50 \%$. Os sinais clínicos se intensificavam durante o dia e eram diretamente proporcionais à elevação da temperatura ambiental. Os achados de necropsia em um bovino que foi eutanasiado, incluíram leve enfisema pulmonar, principalmente na região dorsal dos lobos pulmonares diafragmáticos. Histologicamente havia moderada hipertrofia da musculatura lisa dos bronquíolos e ruptura de septos alveolares formando cotos alveolares em clava. Nos três estabelecimentos onde ocorreram os surtos, escleródios de $C$. purpurea foram observados nas amostras de ração fornecida aos bovinos. Os animais afetados recuperaram-se após aproximadamente 60 
dias da retirada da ração contaminada. O diagnóstico baseouse em dados epidemiológicos, sinais clínicos, na presença de escleródios de $C$. purpurea na ração fornecida aos animais, nos achados de necropsia e na histopatologia. A patogenia e o quadro clínico-patológico observados são discutidos e comparados com outros relatos dessa enfermidade.

TERMOS DE INDEXAÇÃO: Síndrome distérmica, hipertermia, Claviceps purpurea, micotoxicoses, doenças de bovinos.

\section{INTRODUÇÃO}

Em bovinos, a intoxicação por ergoalcalóides produzidos por Claviceps purpurea pode ter quatro apresentações clínicas: (1) síndrome distérmica (SD) (Alvariza et al. 1987, Jessep et al. 1987, Peet et al. 1991, Scrivener \& Bryden 1993, Schneider et al. 1996); (2) gangrena seca das extremidades (Woods et al. 1966, Burfening 1973); (3) forma convulsiva (Riet-Correa 1993) e (4) abortos (Appleyard 1986). A SD tende a ocorrer em estações com temperaturas elevadas (Alvariza et al. 1987, Peet et al. 1991, Schneider et al. 1996), enquanto a forma gangrenosa tipicamente ocorre em temperaturas ambientais mais baixas (Burfening 1973). Assim, a ocorrência simultânea dessas duas formas, em condições espontâneas, é rara.

A SD (também denominada hipertermia) está associada à ingestão de alcalóides do ergot presentes em sementes de plantas infectadas com os escleródios do fungo $C$. purpurea (Alvariza et al. 1987, Jessep et al. 1987, Peet et al. 1991, Scrivener \& Bryden 1993, Schneider et al. 1996). Essa toxicose tem sido também, observada associada ao consumo da gramínea Festuca sp contaminada pelo fungo endofítico Neotyphodium (Acremonium) coenophialum que também produz ergoalcalóides (Paterson et al. 1995, Browning Jr et al. 1998b).

Na SD associada à intoxicação por $C$. purpurea são afetados bovinos de leite (Alvariza et al. 1987, Jessep et al. 1987, Schneider et al. 1996) e de corte mantidos em pastagem (Scrivener \& Bryden 1993, Schneider et al. 1996) ou sob confinamento (Jessep et al. 1987, Peet et al. 1991). Os surtos ocorrem no verão, outono (Alvariza et al. 1987, Peet et al. 1991, Schneider et al. 1996) ou primavera (Alvariza et al. 1987), afetando de 60 a $100 \%$ do rebanho (Jessep et al. 1987, Peet et al. 1991, Schneider et al. 1996). Os sinais clínicos são observados duas a três semanas (Jessep et al. 1987, Peet et al. 1991, Scrivener \& Bryden 1993, Schneider et al. 1996) após a introdução dos animais em pastagens infestadas (Alvariza et al. 1987, Scrivener \& Bryden 1993, Schneider et al. 1996) ou fornecimento de ração (Alvariza et al. 1987, Jessep et al. 1987, Peet et al. 1991, Schneider et al. 1996) ou feno (Alvariza et al. 1987, Schneider et al. 1996) contaminados pelos escleródios de $C$. purpurea.

Sinais clínicos incluem anorexia (Jessep et al. 1987, Peet et al. 1991), diarréia (Alvariza et al. 1987), descarga nasal clara (Peet et al. 1991), aumento da frequiência respiratória (Alvariza et al. 1987, Peet et al. 1991) e salivação excessiva (Alvariza et al. 1987, Jessep et al. 1987, Peet et al. 1991, Schneider et al. 1996). Os bovinos afetados permanecem com a boca aberta e a língua para fora da cavidade oral (Jessep et al. 1987, Schneider et al. 1996). A temperatura corporal ele- va-se para $40-42^{\circ} \mathrm{C}$ (Jessep et al. 1987 , Peet et al. 1991 , Scrivener \& Bryden 1993, Schneider et al. 1996) e há polidipsia (Schneider et al. 1996). Os sinais clínicos de dispnéia acentuam-se com o esforço físico (Jessep et al. 1987, Scrivener \& Bryden 1993, Schneider et al. 1996).

Bovinos afetados tendem a procurar sombra (Alvariza et al. 1987, Jessep et al. 1987, Scrivener \& Bryden 1993, Schneider et al. 1996) ou permanecer dentro d'água (Alvariza et al. 1987, Jessep et al. 1987, Peet et al. 1991, Schneider et al. 1996). A intensidade dos sinais clínicos varia de acordo com a temperatura ambiental (Jessep et al. 1987), sendo maior durante as horas mais quentes $\left(35^{\circ} \mathrm{C}\right)$ do dia (Jessep et al. 1987, Schneider et al. 1996). Muitos bovinos acometidos aparentemente se recuperaram nos dias frios, recidivando o quadro clínico nos dias quentes (Schneider et al. 1996).

Bovinos afetados por SD podem também apresentar anorexia parcial (Alvariza et al. 1987, Scrivener \& Bryden 1993), baixo ganho de peso (Alvariza et al. 1987, Peet et al. 1991) e emagrecimento (Schneider et al. 1996). Nos casos crônicos, descreve-se pelagem longa e sem brilho (Alvariza et al. 1987, Schneider et al. 1996). O crescimento retardado e a pelagem arrepiada e desvitalizada são mais evidentes em animais jovens (Schneider et al. 1996). Em bovinos de leite há redução na produção de leite (Alvariza et al. 1987, Jessep et al. 1987, Schneider et al. 1996). Experimentalmente, observa-se diminuição nas concentrações de prolactina sérica (Ross et al. 1989, Schneider et al. 1996).

Após a interrupção do consumo da alimentação contaminada, a recuperação dos animais afetados é gradual a partir de 2 semanas após a suspensão do alimento contaminado. A recuperação completa ocorre em 2 a 6 meses (Peet et al. 1991, Schneider et al. 1996).

Níveis de 0,02 a $0,8 \%$ de escleródios de $C$. purpurea na ração e contaminação de $25 \%$ das sementes nas pastagens são considerados tóxicos. Entretanto, a toxidez dependerá do tipo e da quantidade de ergoalcalóides presentes (Appleyard 1986, Alvariza et al. 1987, Jessep et al. 1987, Ross et al. 1989, Peet et al. 1991, Schneider et al. 1996).

O ciclo evolutivo de $C$. purpurea no ambiente tem sido bem documentado (Lacey 1991). Escleródios de C. purpurea que caem no chão ou são acidentalmente semeados permanecem no solo durante o outono e inverno. Na primavera, a umidade é ideal para a germinação dos escleródios de $C$. purpurea e, subseqüentemente, para a liberação de ascosporos no ambiente. A germinação dos escleródios ocorre na primavera produzindo ascosporos filamentosos. A disseminação dos ascosporos se dá pelo ar. Os ascosporos infectam a inflorescência, substituindo o ovário da planta, por uma massa micelial brancacenta que produz um líquido semelhante ao mel; esse estágio é denominado conidial ou Sphacelia. Os conídios são produzidos em grande número nesse líquido doce e viscoso atrativo aos insetos, facilitando assim a disseminação e a contaminação para outras inflorescências. Os conídios podem também ser disseminados pela chuva e, talvez, por animais em pastoreio. $O$ conídio aumenta de tamanho, escurece, torna-se rígido e, ao final, transforma-se em um escleródio referido como ergot (Coppock et al. 1989, Lacey 1991). 
Relatam-se aqui três surtos de intoxicação por $C$. purpurea, manifestada sob a forma de hipertermia em bovinos de leite durante o verão de 1999-2000 em três estabelecimentos no Estado do Rio Grande do Sul. Essa forma de intoxicação pelos ergoalcalóides de $C$. purpurea não tinha sido ainda documentada no Brasil.

\section{MATERIAL E MÉTODOS}

Os dados epidemiológicos e os sinais clínicos da doença foram obtidos com os veterinários locais e através de visita ao Estabelecimento 1. Nessa propriedade, um bovino afetado foi eutanasiado e necropsiado. Fragmentos de diversos órgãos e o encéfalo inteiro foram colhidos e processados para exame histológico. Amostras de sangue de três animais foram colhidos em EDTA para eritrograma completo. Swabs nasais de três bovinos afetados e fragmentos de pulmão do animal necropsiado foram processados para inoculação em cultivo celular de células MDBK para isolamento de vírus respiratório sincicial bovino (BRSV). Amostras de ração dos três estabelecimentos foram examinadas para verificar a presença de escleródios de $C$. purpurea.

\section{RESULTADOS}

Os três surtos ocorreram em estabelecimentos de bovinos de leite localizados nos municípios gaúchos de Boa Vista do Buricá (Estab. 1), Três de Maio (Estab. 2) e Maurício Cardoso (Estab. 3). De um total de 66 bovinos, trinta e sete (56\%) foram afetados após o consumo de ração contaminada por $C$. purpurea. Não ocorreram mortes espontâneas. Nos Estabelecimentos 1 e 3 a ração era fornecida pela cooperativa local e no Estabelecimento 2 era formulada pelo produtor.

Estabelecimento 1. Vinte e oito bovinos (70\%) de um total de 40 bovinos holandeses foram afetados. Os animais adoeceram 15 a 20 dias, após a introdução de um novo lote de ração. Essa suplementação alimentar correspondia a $30 \%$ da alimentação dos animais e consistia em aveia, azevém, farelo de soja e milho. Eram fornecidos até $9 \mathrm{~kg}$ de ração/dia/animal. Além da ração, os bovinos recebiam silagem e sorgo forrageiro. Clinicamente, havia salivação intensa, respiração ofegante com a boca aberta e taquipnéia (Fig. 1). Em alguns casos a língua era mantida fora da cavidade oral e o pescoço esticado. Os sinais clínicos eram mais acentuados durante o dia, nos dias mais quentes e nas horas do dia com temperatura mais elevada. Os animais afetados procuravam frequientemente a sombra ou permaneciam dentro d'água. A temperatura era superior a $40^{\circ} \mathrm{C}$ e os pêlos, longos, arrepiados e sem brilho. Alguns bovinos apresentavam claudicação e dificuldade de locomoção. Houve diminuição no consumo de alimento em 20 a 30\% e perda de peso. A diminuição na produção de leite foi de 40 a $50 \%$, chegando a $95 \%$ em alguns dos animais. Houve dois abortos durante o surto. $\mathrm{O}$ rebanho tinha sido vacinado para rinotraqueíte infecciosa bovina e vírus respiratório sincicial bovino (BRSV). O principal achado de necropsia no bovino eutanasiado foi o de enfisema pulmonar, mais acentuado na região dorsal dos lobos pulmonares diafragmáticos. Histologicamente havia moderada hipertrofia da musculatura lisa dos bronquíolos e rompimen-

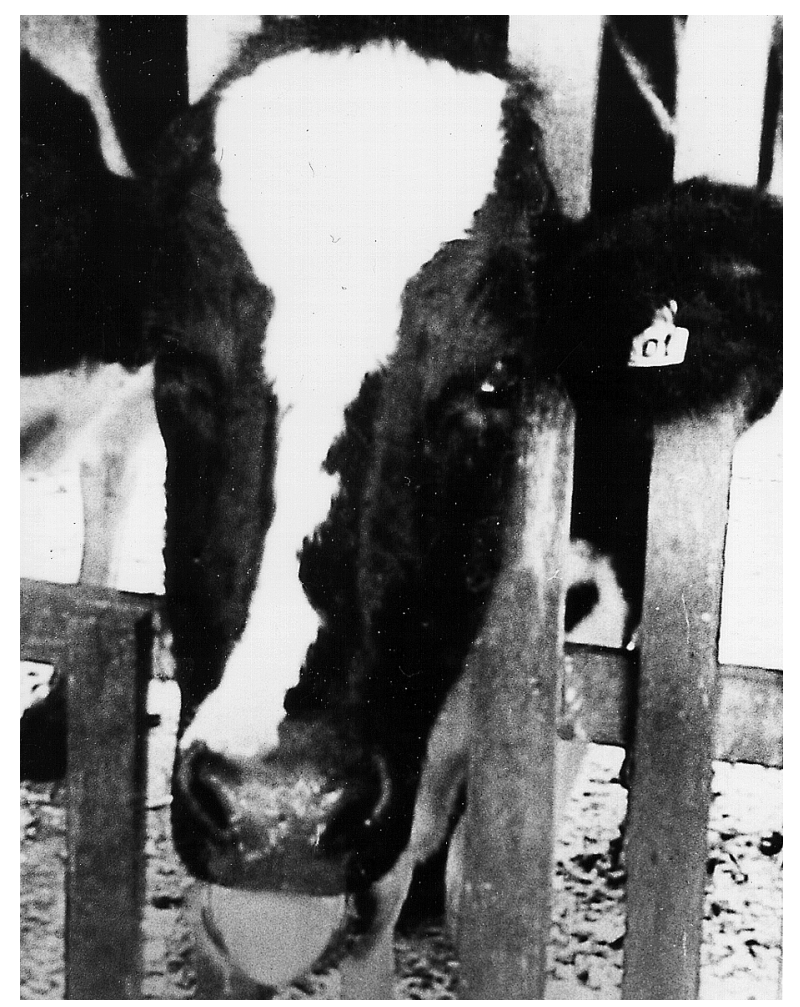

Fig. 1. Vaca afetada por síndrome distérmica mostrando dispnéia, salivação e respiração com boca aberta. Os bovinos afetados também apresentavam febre, procuravam a sombra ou permaneciam dentro d'água.

to de septos alveolares com a formação de bordas em clava. $\mathrm{O}$ eritrograma de três animais afetados estava nos limites normais. Os testes virológicos foram negativos para BRSV. A recuperação dos animais afetados ocorreu até 2 meses após a retirada da ração. A produção de leite retornou aos níveis normais de produtividade do rebanho. No entanto, nos meses subsequientes algumas vacas apresentaram anestro, cio silencioso, infertilidade e baixa concepção.

Estabelecimento 2. Seis $(42,8 \%)$ de um total de 14 bovinos de leite adoeceram 2 meses após a introdução de um novo lote de ração. Essa suplementação alimentar era formulada pelo proprietário e consistia de milho, farelo de soja, farelo de trigo e triticale contaminado com azevém. Os bovinos recebiam em média de 2 a $6 \mathrm{~kg}$ de ração/dia/animal. 0 restante da alimentação era composto de silagem de milho e pastagem de milheto e sorgo. Os sinais clínicos foram semelhantes aos observados nos bovinos do Estabelecimento 1 . Adicionalmente, observou-se aumento no consumo de água. Não foram observadas alterações locomotoras. Houve diminuição no consumo de alimentos de 10 a $20 \%$ e redução na produção de leite em 30 a $40 \%$. A recuperação completa dos animais afetados foi gradativa completando-se em aproximadamente 70 dias. Entretanto, após o surto, quatro animais apresentaram baixa concepção.

Estabelecimento 3. De um total de doze bovinos de leite, três (25\%) foram afetados. O quadro clínico era semelhan- 
te aos observados nos Estabelecimentos 1 e 2. Esses animais recebiam até $6 \mathrm{~kg}$ de ração/dia/animal. A ração era constituída de aveia, azevém, farelo de soja e farelo de milho. $O$ restante da alimentação era composto de silagem de milho e pastagem de milheto e de sorgo. Um animal apresentou claudicação. $\mathrm{O}$ consumo de alimentos e a produção de leite pelos animais afetados diminuíram respectivamente 10 a $20 \%$ e 30 a $40 \%$. Houve aumento no consumo de água. Os sinais clínicos foram observados após cerca de 3 meses da introdução de nova ração na dieta dos bovinos. A recuperação dos animais ocorreu em aproximadamente 60 dias após a retirada da ração-problema. Anestro e baixa concepção foram observados nesses animais após o surto.

Análise da ração. Nas amostras de ração dos três surtos foram encontrados escleródios de C. purpurea (Fig. 2). Esses

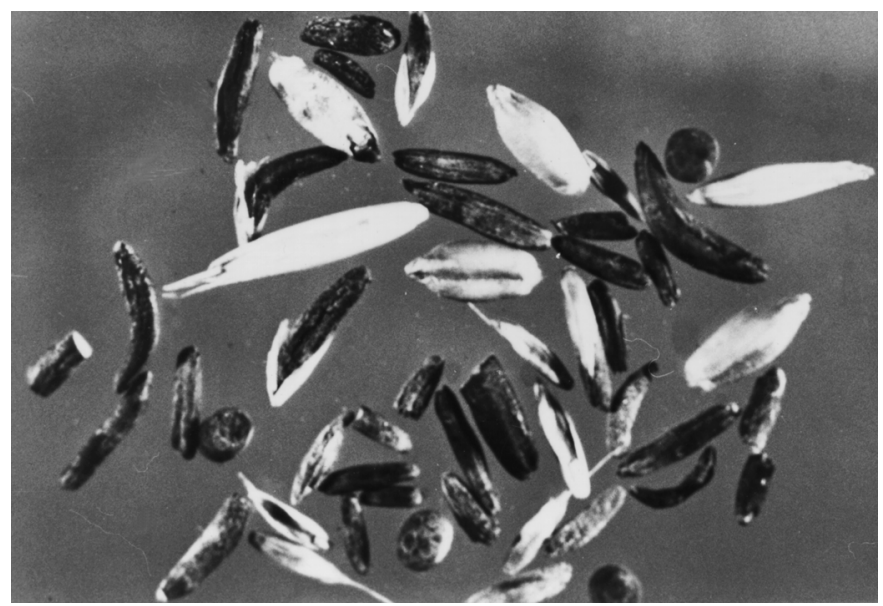

Fig. 2. Amostra da ração usada para alimentação dos bovinos em uma das três propriedades onde ocorreram os surtos de síndrome distérmica. Observam-se vários escleródios de Claviceps purpurea misturados a grãos de cereais.

eram caracterizados por estruturas duras, escuras e curvas medindo aproximadamente $1,0 \mathrm{~cm}$ de comprimento. Fragmentos de escleródios também estavam presentes nessas rações.

\section{DISCUSSÃO}

O diagnóstico de síndrome distérmica (SD) associada à intoxicação por $C$. purpurea em bovinos baseou-se nos dados epidemiológicos, sinais clínicos, na presença de escleródios de $C$. purpurea na ração fornecida aos animais, nos achados de necropsia e na histopatologia.

$\mathrm{Na}$ intoxicação por $C$. purpurea em bovinos, as diversas formas de apresentação clínica podem, ocasionalmente, ocorrer num mesmo rebanho ou em um mesmo animal. Entretanto, a forma convulsiva e a forma gangrenosa da doença raramente são observadas no mesmo surto (Burfening 1973). Na forma gangrenosa, tem sido descrito diminuição na produção de leite (Woods et al. 1966), aumento da temperatura corporal (Burfening 1973, Coppock et al. 1989), salivação e diarréia (Coppock et al. 1989). Esses sinais clínicos também têm sido observados na SD. De forma semelhante, gangrena das extremidades tem sido relatada em alguns casos naturais de SD (Alvariza et al. 1987).

Tem sido sugerido que os efeitos dos alcalóides do ergot resultam de suas ações como agonistas ou antagonistas parciais nos receptores adrenérgicos, dopaminérgicos e triptaminérgicos. A ação farmacológica dessas micotoxinas varia de acordo com o tipo de alcalóide, quantidade ingerida, espécie animal e tecido-alvo (Peroutka 1996). A toxicidade de escleródios de $C$. purpurea é variável e a proporção entre os diversos alcalóides pode diferir consideravelmente (Lacey 1991). Ergotamina, ergostina, ergosina, ergocristina, ergocriptina e ergocornina são os principais alcalóides de $C$. purpurea (Lacey 1991, Schneider 1996).

Nos surtos de síndrome distérmica em bovinos descritos neste relato, a procura pela sombra, a permanência dentro d'água e a respiração ofegante são interpretados como tentativas que os bovinos afetados fazem para reduzir a temperatura corporal. Respiração ofegante, referida como polipnéia térmica, corresponde a um dos meios de perda de calor pela evaporação em animais domésticos. Em bovinos, polipnéia térmica usualmente ocorre em animais que apresentam temperatura retal superior a $40^{\circ} \mathrm{C}$ (Anderson 1988).

A sudorese é o principal mecanismo de perda de calor por evaporação em bovinos (Anderson 1988). A dissipação de calor através da superfície cutânea pode ocorrer pelas formas não evaporativas (conveç̧ão e condução) ou pela forma evaporativa (sudorese). Essas formas de perda de calor dependem do fluxo sangüíneo na pele. Na pele de bovinos, há uma estreita associação anatômica entre a rede capilar cutânea e as glândulas sudoríparas. Assim, a diminuição do fluxo sanguiíneo para a pele dificulta a transferência térmica e a produção de suor (Finch 1986). A ocorrência de hipertermia em bovinos associada à ingestão de ergoalcalóides ocorre devido à ação vasoconstritora periférica dessas micotoxinas que reduz o fluxo do sangue para a pele e a perda de calor na periferia (Osborn et al. 1992, Paterson et al. 1995, Browning Jr. et al. 1998b). Essa hipótese é consubstanciada pelo fato de que a administração experimental de ergoalcalóides a bovinos mantidos em temperaturas de $32^{\circ} \mathrm{C}$ reduz a evaporação da pele (Aldrich et al. 1993). A administração de ergotamina, o principal ergoalcalóide de $C$. purpurea, para bovinos mantidos em temperatura de $30^{\circ} \mathrm{C}$ promove $o$ aumento da frequiência respiratória, decréscimo na temperatura da pele e elevação da temperatura retal (Osborn et al. 1992, Browning Jr. \& Leite-Browning 1997). $O$ aumento na temperatura retal também tem sido associado ao aumento na produção de calor pela estimulação das oxidases de função mista hepáticas, envolvidos na detoxificação dos alcalóides do ergo (Zanzarali et al. 1989).

A administração experimental de alcalóides do ergot a bovinos por períodos curtos provoca aumento nas concentrações de triiodotironina (T3), tiroxina (T4), cortisol (Browning Jr et al. 1998b) e hormônio do crescimento (GH) (Browning Jr et al. 1997). Esses experimentos sugerem que alterações nas concentrações plasmáticas de cortisol, T3 e T4 tenham influência nas funções metabólicas e termor- 
regulatórias (Browning Jr et al. 1998b). No entanto, bovinos expostos naturalmente aos alcalóides do ergot por períodos prolongados não apresentam variações nas concentrações plasmáticas de T3, T4 e cortisol (Aldrich et al. 1993).

Experimentalmente, bovinos que receberam doses elevadas de escleródios de $C$. purpurea, por períodos prolongados em temperaturas superiores a $22^{\circ} \mathrm{C}$, apresentaram um quadro típico de ergotismo gangrenoso acompanhado de sinais clínicos de SD (Alvariza et al. 1987). Nos Estabelecimentos 1 e 3 alguns animais apresentaram claudicação. Todavia, não foram observadas lesões de gangrena das extremidades. Esse distúrbio locomotor pode ser atribuído a lesões podais e músculo-esqueléticas. Em bovinos, cerca de 90\% das causas de claudicação são originárias de problemas nos cascos. O manejo e a alimentação de bovinos de leite favorecem o desenvolvimento de claudicações dessa origem (Rebhun 1995).

Há relatos de intoxicação espontânea por alcalóides de $C$. purpurea em bovinos que cursam com aborto, agalactia e retenção de placenta (Rankin 1965, Mantle \& Gunner 1965, Appleyard 1986, Rivero et al. 1989). A ocorrência desses distúrbios reprodutivos tem sido atribuída à ação dos ergoalcalóides (Appleyard 1986). É oportuno mencionar que a etiologia dos abortos em bovinos é multifatorial e incluem agentes infecciosos, tóxicos, nutricionais e ambientais. Assim, virtualmente qualquer elemento que comprometa o estado de saúde geral dos animais gestantes pode resultar em aborto. No presente relato, os abortos ocorridos no Estabelecimento 1 não foram investigados quanto à etiologia específica através de exames laboratoriais complementares o que torna a associação dos abortos ao quadro de intoxicação apenas especulativa.

Problemas reprodutivos semelhantes aos descritos aqui têm sido observados na intoxicação por alcalóides do ergot em bovinos após a aparente recuperação clínica. Na SD têm sido descritos cio silencioso, anestro, baixa concepção e infertilidade. Além disso, há aumento da duração do período entre partos e maior número de inseminações por concepção (Schneider et al. 1996) ao passo que na forma gangrenosa já foi observada repetição de cio (Woods et al. 1966). De forma semelhante, bovinos mantidos em pastagens de festuca contaminada com o fungo Neotyphodium coenophialum apresentam taxas de concepção reduzidas (Paterson et al. 1995). Os mecanismos patogenéticos envolvidos nos distúrbios reprodutivos observados nesta micotoxicose não estão completamente esclarecidos. Há evidências experimentais de que a administração endovenosa de ergotamina e de ergonovina por curtos períodos de tempo em bovinos diminui a concentração de LH (hormônio luteinizante) (Browning Jr et al. 1997, Browning Jr et al. 1998a) e aumenta as concentrações de $\mathrm{PGF}_{2} \mathrm{a}$, hormônios que interferem na função reprodutiva (Browning Jr et al. 1998a).

A diminuição na produção de leite observada nos casos deste relato é descrita na intoxicação por alcalóides do ergot (Woods et al. 1966, Alvariza et al. 1987, Jessep et al. 1987, Paterson et al. 1995, Schneider et al. 1996) e tem sido explicada pela diminuição nas concentrações séricas de prolactina em casos naturais (Schneider et al. 1996) e experimentais (Ross et al. 1989, Schneider et al. 1996) de intoxicação por escleródios de $C$. purpurea. $O$ principal fator inibidor da liberação de prolactina pela adeno-hipófise é a dopamina (Neill \& Nagy 1994). Sugere-se que a atividade dos alcalóides do ergot semelhante à dopamina seja responsável pela diminuição da prolactina sérica (Paterson et al. 1995).

Neste estudo, foi observado enfisema na face dorsal dos lobos diafragmáticos dos pulmões e hiperplasia da túnica da musculatura lisa de bronquíolos. Achados semelhantes têm sido descritos por outros autores (Schneider et al. 1996). No enfisema alveolar, lesões microscópicas incluem ruptura de septos alveolares e formação de bordas em clava (Jones et al. 1997). Sugere-se que essas alterações podem ser atribuídas a polipnéia térmica de duração prolongada. Alterações histológicas na parede de vasos descritas nos casos de SD e de ergotismo gangrenoso em bovinos (Coppock et al. 1989, Schneider et al. 1996) não foram observadas nos bovinos deste relato.

Os sinais clínicos respiratórios manifestados na intoxicação por ergoalcalóides podem ser confundidos com os observados em doenças respiratórias de bovinos de etiologia infecciosa, parasitária ou tóxica, particularmente naquelas regiões onde a ocorrência da micotoxicose é desconhecida. Assim, a SD deve ser diferenciada das enfermidades que afetam o trato respiratório de rebanhos bovinos sob a forma de surtos e que cursam com sinais de insuficiência respiratória e hipertermia. Na nossa região, as seguintes condições devem ser incluídas no diagnóstico diferencial: (1) complexo respiratório bovino (pneumonia enzoótica), que compreende o vírus sincicial respiratório bovino (BRSV), herpesvírus bovino do tipo 1 (vírus da rinotraqueíte infecciosa bovina), vírus da parainfluenza-3 (PI-3) e diversos microrganismos, como por exemplo, Pasteurella sp e Chlamydia sp isoladamente ou em conjunto, (2) parasitismo por Dictyocaulus viviparus (pneumonia verminótica), (3) pneumonia intersticial atípica (edema e enfisema pulmonar, "fog fever") e (4) "golpe de calor" ("heat stroke"), síndrome de etiologia multifatorial usualmente associada a erros de manejo em confinamento de bovinos (Schild et al. 1986, Radostits et al. 1994, Riet-Correa et al. 1998).

Nos surtos de intoxicação por escleródios de $C$. purpurea, a fonte de contaminação usualmente é a pastagem ou o feno contaminado com azevém sementado (Woods et al. 1966, Appleyard 1986, Scrivener \& Bryden 1993, Schneider et al. 1996). Alternativamente, esses escleródios podem vir de grãos contaminados com sementes de azevém infectadas por C. purpurea (Jessep et al. 1987, Schneider et al. 1996). As espécies de azevém envolvidas nos surtos desta micotoxicose em geral são azevém perene (Lolium perenne) (Woods et al. 1966) e anual (Lolium rigidum) (Jessep et al. 1987, Scrivener \& Bryden 1993, Schneider et al. 1996). No Rio Grande do Sul, $C$. purpurea tem sido observado na primavera infectando Lolium multiflorum (Riet-Correa 1993). Em nosso estudo, acredita-se que a fonte de contaminação nos três surtos tenha sido o azevém contaminado com $C$. purpurea. Outras espécies de fungos endofíticos podem produzir alcalóides do ergot 
à semelhança do que ocorre em Neotyphodium coenophialum (Lacey 1991). Nos surtos aqui descritos, não foi realizada pesquisa micológica de fungos endofíticos nos componentes das rações fornecidas aos bovinos. Assim, a presença simultânea de $C$. purpurea e de fungos endofíticos produtores de ergoalcalóides não pode ser completamente descartada.

Não há tratamento eficaz para a síndrome distérmica associada à ingestão de ergoalcalóides. Uma vez diagnosticada a enfermidade no rebanho, a fonte de contaminação deve ser imediatamente retirada da alimentação dos animais. A recuperação dos bovinos doentes pode variar de 2 a 6 meses da suspensão da suplementação alimentar contaminada. Para prevenir a ocorrência da micotoxicose, deve-se evitar o uso de grãos e pastagens contaminados pelo fungo. Procedimentos adequados de ceifamento evitam o surgimento de inflorescência. $O$ aramento profundo do solo enterra os escleródios e impede a contaminação das pastagens (Lacey 1991).

Nos Estados Unidos, estima-se que 609 milhões de dólares são perdidos anualmente na bovinocultura de corte pela manutenção desses animais em pastagens de festuca contaminadas com Neotyphodium coenophialum (Paterson et al. 1995). No presente relato, os maiores prejuízos econômicos sofridos pelos pequenos produtores de leite que empregaram rações contaminadas por $C$. purpurea ocorreram por queda na produção leiteira de seus rebanhos e pela lenta recuperação dos animais afetados.

Agradecimentos.- Os autores são agradecidos aos Médicos Veterinários Carlos Diesel, de Boa Vista do Buricá, e Álvaro Callegaro, de Horizontina, pelo auxílio na obtenção dos históricos clínicos.

\section{REFERÊNCIAS}

Aldrich C.G., Paterson J.A., Tate J.L. \& Kerley M.S. 1993. The effects of endophyte-infected tall fescue consumption on diet utilization and thermal regulation in cattle. J. Anim. Sci. 71(1):164-170.

Alvariza F.R., Perdomo E., Capano F., Collazo S. \& Morón C. 1987. Cuadros de asoleamiento en bovinos asociados al hongo Claviceps purpurea. IV Congr. Nac. Veterinaria, Montevideo, Uruguai. (Mimeografado)

Anderson B.E. 1988. Regulação da temperatura e fisiologia ambiental, p. 623629. In: Swenson M.J. Dukes (ed.) Fisiologia dos Animais Domésticos. $10^{\mathrm{a}}$ ed., Guanabara-Koogan, Rio de Janeiro. 799 p.

Appleyard W. T. 1986. Outbreak of bovine abortion attributed to ergot poisoning. Vet. Rec. 118(2):48-49.

Browning Jr R. \& Leite-Browning M.L. 1997. Effect of ergotamine and ergonovine on thermal regulation and cardiovascular function in cattle. J. Anim. Sci. 75(1):176-181.

Browning Jr R., Thompson F.N., Sartin J.L. \& Leite-Browning M.L. 1997. Plasma concentrations of prolactin, growth hormone, and luteinizing hormone in steers administered ergotamine or ergonovine. J. Anim. Sci. 75(3):796-802.

Browning Jr R., Schrick F.N., Thompson F.N. \& Wakefield Jr T. 1998a. Reproductive hormonal responses to ergotamine and ergonovine in cows during the luteal phase of the estrous cycle. J. Anim. Sci. 76(5):1448-1454.

Browning Jr R., Leite-Browning M.L., Smith H.M. \& Wakefield Jr T. 1998b. Effect of ergotamine and ergonovine on plasma concentrations of thyroid hormones and cortisol in cattle. J. Anim. Sci. 76(6):1644-1650.

Burfening P.J. 1973. Ergotism. J. Am. Vet. Med. Assoc. 163(11):1288-1290.

Coppock R.W., Mostrom M.S., Simon J., McKenna D.J., Jacobsen B. \& Szlachta
H.L. 1989. Cutaneous ergotism in a herd of dairy calves. J. Am. Vet. Med. Assoc. 194(4):549-551.

Finch V.A. 1986. Body temperature in beef cattle: its control and relevance to production in the tropics. J. Anim. Sci. 62(2):531-542.

Jessep T.M., Dent C.H.R., Kemp J.B., Christie B., Ahrens P.J., Burgess L.W. \& Bryden W.L. 1987. Bovine idiopathic hypertermia. Aust. Vet. J. 64(11):353-354.

Jones T.C., Hunt R.D. \& King N.W. 1997. Veterinary Pathology. 6th ed. Williams \& Wilkins, Baltimore, p. 947-973.

Lacey J. 1991. Natural occurrence of mycotoxins in growing and conserved forage crops, p.363-397. In: Smith J.E. \& Henderson R.S. (ed.) Mycotoxins and Animal Foods. CRC, Boca Raton.

Mantle P.G. \& Gunner, D.E. 1965. Abortions associated with ergotised pastures. Vet. Rec. 77(30):885-886.

Neill J.D. \& Nagy G.M. 1994. Prolactin secretion and its control, p. 18331860. In: Knobil E. \& Neill, J.D. (ed.) The Physiology of Reproduction. 2nd ed. Raven, New York.

Osborn T.G., Schmidt S.P., Marple D.N., Rahe C.H. \& Steenstra J.R. 1992. Effect of consuming fungus-infected and fungus-free tall fescue and ergotamine tartrate on selected physiological variables of cattle in environmentally controlled conditions. J. Anim. Sci. 70(8):2501-2509.

Paterson J., Forcherio C., Larson B., Samford M. \& Kerley M. 1995. The effects of fescue toxicosis on beef cattle productivity. J. Anim. Sci. 73(3):889-898.

Peet R.L., McCarthy M.R. \& Barbetti M.J. 1991. Hyperthermia and death in feedlot cattle associated with the ingestion of Claviceps purpurea. Aust. Vet. J. 68(3): 12 .

Peroutka S.J. 1996. Fármacos eficazes no tratamento da enxaqueca, p. 354366. In: Hardman G.J. \& Limbird L.E. (ed.) Goodman \& Gilman's. As bases Farmacológicas da Terapêutica. $9^{\underline{a}}$ ed. McGraw-Hill, Rio de Janeiro.

Radostits O.M., Blood D.C. \& Gay C.C. 1994. Veterinary Medicine. A textbook of the diseases of cattle, sheep, pigs, goats and horses. 8th ed. Baillière Tindall, London, p. 38-106.

Rankin J.E.F. 1965. Abortions associated with ergotised pastures. Vet. Rec. 77(31):911-912.

Rebhun W.C. 1995. Diseases of Dairy Cattle. Williams \& Wilkins, Philadelphia, p. 369-406.

Riet-Correa F. 1993. Micotoxicoses que produzem ergotism, p. 227-245. In: Riet-Correa F., Méndez M.C. \& Schild A.L. (ed.) Intoxicações por Plantas e Micotoxicoses em Animais Domésticos. Editorial Hemisfério Sul do Brasil, Pelotas.

Riet-Correa F., Schild A.L. \& Méndez M.C. 1998. Doenças de Ruminantes e Eqüinos. Editora Universitária, UFPel, Pelotas. 651 p.

Rivero R., Quintana, S., Féola, R., Haedo, F. 1989. Principales enfermedades diagnosticadas em el área de influencia del laboratório de diagnostico regional noroeste del C. I. Vet. "Miguel Rubino". XVII Jornadas Uruguayas de Buiatria, Paisandú, Uruguai. Seção I, p. 1- 73. (Resumo)

Ross A.D., Bryden W.L., Barau W. \& Burgess L.W. 1989. Induction of heat stress in beef cattle by feeding the ergots of Claviceps purpurea. Aust. Vet. J. 66(8):247-249.

Schneider D.J., Miles C.O., Garthwaite I., Van Halderen A., Wessels J.C. \& Lategan H.J. 1996. First report of field outbreaks of ergot-alkaloid toxicity in South Africa. Onderstepoort J. Vet. Res. 63 (2): 97-108.

Schild A.L., Riet-Correa F., Méndez M.C., Ribeiro W.N.L. \& Meireles M.C.A. 1986. Laboratório Regional de Diagnóstico, Doenças Diagnosticadas no Ano de 1985. Editora Universitária, UFPel, Pelotas, 30p.

Scrivener C.J. \& Bryden W.L. 1993. Hyperthermia in cattle grazing annual ryegrass. N.Z. Vet. J. 41(4):215.

Woods A.J., Jones J.B. \& Mantle P.G. 1966. An outbreak of gangrenous ergotism in cattle. Vet. Rec. 78(22):742-749.

Zanzalari K.P., Heitmann R.N., McLaren J.B. \& Fribourg H.A. 1989. Effects of endophite-infected fescue and cimetidine on respiration rates, rectal temperatures and hepatic mixed function oxidase activity as measured by hepatic antipyrine metabolism in sheep. J. Anim. Sci. 67(12):3370-3378. 